\title{
Electrical stimulation of the common peroneal nerve and its effects on the relationship between corticomuscular coherence and motor control in healthy adults
}

\author{
Tadaki Koseki', Daisuke Kudo ${ }^{1}$, Natsuki Katagiri' ${ }^{1}$ Shigehiro Nanba ${ }^{1}$, Mitsuhiro Nito ${ }^{2}$, Shigeo Tanabe ${ }^{3}$ and
} Tomofumi Yamaguchi ${ }^{1,4^{*}}$ (D)

\begin{abstract}
Background: Sensory input via neuromuscular electrical stimulation (NMES) may contribute to synchronization between motor cortex and spinal motor neurons and motor performance improvement in healthy adults and stroke patients. However, the optimal NMES parameters used to enhance physiological activity and motor performance remain unclear. In this study, we focused on sensory feedback induced by a beta-band frequency NMES ( $\beta$-NMES) based on corticomuscular coherence (CMC) and investigated the effects of $\beta$-NMES on CMC and steady-state of isometric ankle dorsiflexion in healthy volunteers. Twenty-four participants received $\beta$-NMES at the peak beta-band CMC or fixed NMES (f-NMES) at $100 \mathrm{~Hz}$ on different days. NMES was applied to the right part of the common peroneal nerve for $20 \mathrm{~min}$. The stimulation intensity was $95 \%$ of the motor threshold with a pulse width of $1 \mathrm{~ms}$. The beta-band CMC and the coefficient of variation of force (Force CV) were assessed during isometric ankle dorsiflexion for $2 \mathrm{~min}$. In the complementary experiment, we applied $\beta$-NMES to 14 participants and assessed beta-band CMC and motor evoked potentials (MEPs) with transcranial magnetic stimulation.
\end{abstract}

Results: No significant changes in the means of beta-band CMC, Force CV, and MEPs were observed before and after NMES conditions. Changes in beta-band CMC were correlated to (a) changes in Force CV immediately, at 10 min, and at 20 min after $\beta$-NMES (all cases, $p<0.05$ ) and (b) changes in MEPs immediately after $\beta$-NMES $(p=0.01)$. No correlations were found after f-NMES.

Conclusions: Our results suggest that the sensory input via NMES was inadequate to change the beta-band CMC, corticospinal excitability, and voluntary motor output. Whereas, the $\beta$-NMES affects the relationship between changes in beta-band CMC, Force CV, and MEPs. These findings may provide the information to develop NMES parameters for neurorehabilitation in patients with motor dysfunction.

Keywords: Neuromuscular electrical stimulation, Beta-band stimulation, Sensory input, Corticomuscular coherence, Isometric contraction, Corticospinal excitability

*Correspondence: t.yamaguchi.ja@juntendo.ac.jp

${ }^{4}$ Department of Physical Therapy, Faculty of Health Science, Juntendo

University, 2-1-1 Hongo, Bunkyo-ku, Tokyo 113-8421, Japan

Full list of author information is available at the end of the article

\section{Introduction}

Motor control is essential for the interactions with the environment. It is heavily dependent on the descending cortical control of the muscles $[1,2]$. Specifically, the developed steady-state forces during daily living (i.e., 
grasping a cup, standing) are controlled by sensorimotor binding through somatosensory feedback to cortex [3]. Therefore, the enhancement of the communication between cortex and muscles may improve motor control that may in turn contribute to the development of rehabilitation strategies for patients who suffer from motor dysfunction.

Corticomuscular coherence (CMC) is a method used to assess synchronization between motor cortex and spinal motor neurons activities [1,3-8]. The magnitude of beta-band CMC $(15-35 \mathrm{~Hz})$ is correlated with the ability to stabilize muscle force output [3]. The beta-band CMC is significantly lower in stroke patients compared with healthy controls $[9,10]$. Alternatively, the enhancement in beta-band CMC is related to motor learning processes $[7,11]$, and motor functional recovery after stroke [12]. Thus, the development of a technique to increase beta-band CMC is expected to enhance motor learning and promote motor recovery in patients with stroke.

Several studies have demonstrated that beta-band CMC is modulated by an afferent input $[6,13-16]$. One possible strategy for the enhancement of CMC is neuromuscular electrical stimulation (NMES). The peripheral sensory inputs via NMES enhanced the activities of circuits in the somatosensory cortex [17-19]. The activation of the somatosensory cortex by NMES indirectly enhances the excitability of primary motor cortex and projections to the spinal cord [19-21]. Therefore, NMES enhances beta-band CMC and motor functional recovery in individuals with stroke [22]. However, another study has reported that NMES enhances gamma-band CMC, but beta-band CMC did not significantly change [23]. These results suggested that the sensory input via NMES may affect CMC and motor performance, although the evidence has been inconclusive. Additionally, prior studies only used fixed NMES frequencies (e.g., $100 \mathrm{~Hz}$ ). To the best of our knowledge, no prior study has focused on the beta-band frequency of NMES based on physiological activity, and the mechanisms underlying these effects still remain unclear.

A previous study reported that transcranial alternating current stimulation (tACS) with stimulus frequency based on individual's beta-band CMC enhances CMC and performance retention [24]. Therefore, we hypothesized that NMES with beta-band CMC frequency changes $\mathrm{CMC}$ and muscle force control. In the present study, we investigated whether NMES corresponding to beta-band CMC frequency can enhance synchronization between motor cortex and spinal motor neurons, and whether it can improve the exerted steady-state of ankle dorsiflexion in healthy individuals.

\section{Methods}

\section{Participants}

Twenty-four healthy volunteers (12 females) aged 21-30 years old, (mean \pm standard deviation: SD, $22 \pm 2$ years) participated in the experiment. None of the participants had any history of neurological and/or musculoskeletal disorders. The number of participants was decided based on a previous study that investigated the modulation of CMC by NMES [23]. All participants were right-foot dominant as confirmed by the foot-dominant test [25]. All participants provided written informed consent before participation. The experimental protocol was approved by the Ethical Review Board of Yamagata Prefectural University of Health Science in Japan and conformed to the ethical standards laid down in the 1964 Declaration of Helsinki.

\section{NMES}

NMES was delivered with a stimulator (SEN-3401, Nihon Kohden, Tokyo, Japan). The first stimulation frequency for NMES was defined as beta-band frequency NMES ( $\beta$-NMES) and was estimated from the peak of the beta-band CMC frequency (mean $\pm S D, 21 \pm 5 \mathrm{~Hz}$ ), while participants performed isometric dorsiflexion tasks before the stimulation. The second stimulation frequency for NMES was defined as the fixed frequency of NMES (f-NMES) and consisted of a train of 10 pulses at $100 \mathrm{~Hz}$ generated every $2 \mathrm{~s}$ [26]. The electrodes were applied to the skin above the right common peroneal nerve (CPN) for $20 \mathrm{~min}$ [27]. The stimulation intensity was $95 \%$ of the motor threshold with a pulse width of $1 \mathrm{~ms}$. The motor threshold was defined such that the minimum stimulation intensity evoked muscle twitch contraction of the tibialis anterior (TA) muscle.

\section{Electroencephalographic (EEG) and electromyographic (EMG) recordings}

The participants sat comfortably on a chair with their feet firmly strapped to a foot plate. EEG and EMG were recorded with $\mathrm{Ag} / \mathrm{AgCl}$ electrodes. Before the attachment of electrodes, the skin was rubbed with an alcohol pad and the skin impedance was kept below $5 \mathrm{k} \Omega$. EEG electrodes were placed at $\mathrm{Cz}$ representing the ankle muscles and at $5 \mathrm{~cm}$ frontal to $\mathrm{Cz}$, according to the international 10-20 system of electrode placement [7]. EMG electrodes were placed over the TA muscle belly in the right lower limb with an inter-electrode distance of $2 \mathrm{~cm}$. EEG and EMG data were simultaneously acquired (Neuropack MEB-2200, Nihon Kohden, Tokyo, Japan), bandpass filtered in the frequency range of $0.5-200 \mathrm{~Hz}$ in the case of EEG and in the range of $5-500 \mathrm{~Hz}$ in the case of EMG. Force signals were recorded with a force transducer (Takei Scientific Instruments Co. Niigata, Japan). 
EEG, EMG, and force data were recorded while the participants performed isometric dorsiflexion to maintain their exerted force as close as possible to the line that corresponded to $10 \%$ of their maximum voluntary contraction (MVC) force for $2 \mathrm{~min}$ [28]. All signals were converted to digital signals at a sample frequency of $5 \mathrm{kHz}$ by analog-to-digital converter with a 16-bit resolution (NI USB-6363, National Instruments, Austin, TX, USA) controlled by the data-logger software LabVIEW2018 (National Instruments Co., TX, USA).

\section{Corticomuscular coherence}

CMC was estimated by EEG and EMG data following Eq. (1) [29]. CMC describes the linear association between EEG and EMG signals at each frequency of interest. It is a measure of phase consistency between signals. CMC estimates are defined over the range $0-1$, where the value of zero indicates no association, and one indicates a perfect association. To calculate CMC, autospectra and cross-spectra were constructed by dividing signals into nonoverlapping segments. Discrete Fourier transforms were then performed on each segment and averaged. CMC was then determined as the squared modulus of the cross-spectrum for the two signals $f_{x y}(j)$, normalized by the product of the two auto-spectra, $f_{x x}(j)$, and $f_{y y}(j)[28]$.

$$
|R x y(j)|^{2}=\frac{|f x y(j)|^{2}}{f x x(j) f y y(j)}
$$

The statistical significance of $\mathrm{CMC}$ estimates was assessed according to an upper 95\% confidence limit.

\section{Force steadiness of ankle dorsiflexion}

The right foot was strapped to the plate with the ankle in the neutral position. Participants were asked to maintain an isometric dorsiflexion at $10 \% \mathrm{MVC}$ for $2 \mathrm{~min}$. The target force line was presented in front of participants with a display. Participants were instructed to follow the target line as closely as possible with the moving red line (realtime dorsiflexion force). It was reported that the betaband CMC was negatively correlated with the coefficient of variation [(standard deviation/mean) $\times 100 \%$ ] of force (Force CV)3. If NMES changes the beta-band CMC, the changes in the beta-band $\mathrm{CMC}$ could be related with the changes in the exerted steady-state of ankle dorsiflexion. To investigate the exerted steadiness of dorsiflexion force during the ankle dorsiflexion task, Force CV was calculated as a measure of the control of the exerted force.

\section{Experimental procedure}

This study employed a randomized crossover design. Participants received $\beta$-NMES and $\mathrm{f}$-NMES on two different days (Fig. 1A). To assess beta-band CMC changes, EEG and EMG were measured before stimulation (pre) and after stimulation at $0 \mathrm{~min}$ (post), $10 \mathrm{~min}$ (post10) and $20 \mathrm{~min}$ (post20). To prevent carry-over effects from the previous NMES condition, washout intervals of 3 days (or more) were included between sessions.

\section{Complementary experiment}

Beta-band CMC reflects the functional coupling between primary motor cortex and spinal motor neurons that related to corticospinal excitability $[9,30]$. Corticospinal excitability was modulated by NMES [27, 31-33]. Therefore, the changes in the beta-band CMC induced by $\beta$-NMES may be related to the changes in corticospinal excitability. To address this question, 14 healthy volunteers (5 females) aged 20-32 years old, (mean \pm SD, $24 \pm 3$ years) received $\beta$-NMES (mean \pm SD, $25 \pm 5 \mathrm{~Hz}$ ) for $20 \mathrm{~min}$ (Fig. 1B). To assess corticospinal excitability, single-pulse transcranial magnetic stimulation was delivered to the primary motor cortex responsible for motor representation of the leg with a figure- 8 coil $\left(\mathrm{D}^{2} 0^{2}\right)$ that was connected to the Magstim 200 (Magstim Company, Whitland, United Kingdom). The optimal coil positioning on the hot spot of the primary motor cortex was identified for the induction of the largest motor evoked potentials (MEPs) amplitudes in the right TA. The stimulation intensity was adjusted to $120 \%$ of the active motor threshold (aMT). The aMT was defined as the minimum

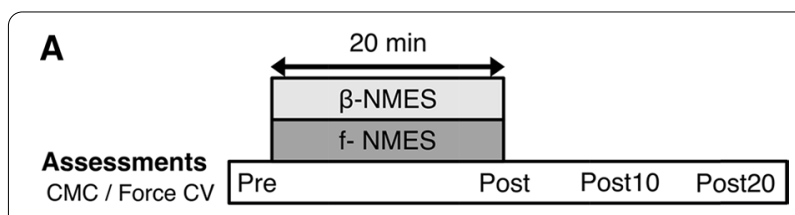

B

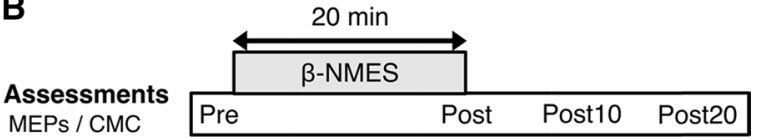

Fig. 1 Experimental paradigm. Time course of main experimental (A) and Complementary experiment (B). In the main experiment, all participants received neuromuscular electrical stimulation (NMES) at two different frequencies as follows: (1) beta-band frequency NMES ( $\beta$-NMES) frequency estimated by the peak of the beta-band corticomuscular coherence (CMC) in the range of $15-35 \mathrm{~Hz}$ and (2) a fixed $100 \mathrm{~Hz}$ NMES (f-NMES) frequency. The beta-band CMC and the coefficient of variation of force (Force CV) were assessed before stimulation (pre) and after stimulation at $0 \mathrm{~min}$ (post), $10 \mathrm{~min}$ (post10), and $20 \mathrm{~min}$ (post20). In the complementary experiment, all participants received $\beta$-NMES. The motor evoked potentials (MEPs) and the beta-band $C M C$ were assessed before stimulation (pre) and after stimulation at $0 \mathrm{~min}$ (post), $10 \mathrm{~min}$ (post10), and $20 \mathrm{~min}$ (post20) 
stimulus intensity that produced $200 \mu \mathrm{V}$ MEPs with a probability of $50 \%$ during isometric contraction at $100 \mu \mathrm{V}$ of TA EMG. Fifteen MEPs were recorded while the participants performed an isometric contraction with $100 \mu \mathrm{V}$ of TA. Before the main assessment, baseline MEPs and CMC were measured to normalize the data. Following a 2-min rest period, MEPs and CMC were assessed pre, post at $0 \mathrm{~min}$ (post), $10 \mathrm{~min}$ (post10), and $20 \mathrm{~min}$ (post20). To minimize the influences of voluntary contraction during the CMC assessment, the MEPs were assessed experimentally before the CMC assessment.

\section{Statistical analysis}

Normality was assessed with the Shapiro-Wilk test. The Wilcoxon signed-rank test was used to compare pre data between the raw value of the beta-band CMC and Force $\mathrm{CV}$. Friedman test was used to investigate the main effect of time (pre, post, post10, and post20) on the raw values of the beta-band CMC and Force CV. Post-hoc tests were performed using the Wilcoxon signed-rank test with Bonferroni adjustments when a significant main effect was found. Spearman's rank correlation was used to define the relationship between the normalized betaband CMC and the Force CV induced by stimulations in assessment time points (post, post10, and post20). The normalized beta-band CMC and normalized Force CV were calculated by the obtained data pre.

In the complementary experiment, the normalized beta-band CMC and normalized MEPs were calculated with the baseline data. Friedman's test was used to investigate the main effect of time (pre, post, post10, and post20) on the normalized MEPs. Post-hoc tests were performed with the Wilcoxon signed-rank test with Bonferroni adjustments when a significant main effect was found. Pearson's product moment correlation was used to investigate the relationship between the normalized MEPs and normalized beta-band CMC at assessment time points after $\beta$-NMES (pre, post, post10, and post20). P-values $<0.05$ indicated statistical significance in all analyses. Statistical analyses were performed with SPSS (version 24.0, IBM Corporation, New York, NY, USA) for Windows.

\section{Results}

The Shapiro-Wilk test showed that the main experimental data on the beta-band $\mathrm{CMC}$, Force $\mathrm{CV}$, normalized beta-band $\mathrm{CMC}$, and normalized Force $\mathrm{CV}$ were not normally distributed.

CMC

The mean raw data $(\mathrm{SD})$ of the beta-band $\mathrm{CMC}$ at pre were 0.07 (0.06) in $\beta$-NMES and $0.06(0.05)$ in f-NMES. No significant difference was observed between $\beta$-NMES and $\mathrm{f}$-NMES conditions at pre (Wilcoxon signed-rank test, $p=0.08)$. No significant main effects were observed on $\beta$-NMES $(p=0.21)$ (Fig. $2 A)$ and f-NMES $(p=0.11)$ (Fig. 2B).

\section{Force CV}

The mean raw data $(\mathrm{SD})$ of the Force $\mathrm{CV}$ at pre were 2.9 (1.3) \% in $\beta$-NMES and $2.7(1.0) \%$ in f-NMES. No significant difference was observed between $\beta$-NMES and f-NMES conditions at pre (Wilcoxon signed-rank test, $\mathrm{p}=0.22$ ). Furthermore, no significant main effects were found on $\beta$-NMES $(p=0.13)$ and $f-N M E S(p=0.74)$.

\section{Relationship between normalized beta-band CMC and Force CV}

Figure 3 shows data plots on the normalized beta-band CMC and normalized Force CV. For $\beta$-NMES condition, there were significant negative correlations at post $(r=-0.54, p=0.01$, Fig. $3 \mathrm{~A})$, post10 $(r=-0.47$, $\mathrm{p}=0.02$, Fig. $3 \mathrm{~B})$, and post $20(\mathrm{r}=-0.42, \mathrm{p}=0.04$, Fig. $3 C$ ). However, the f-NMES condition showed that

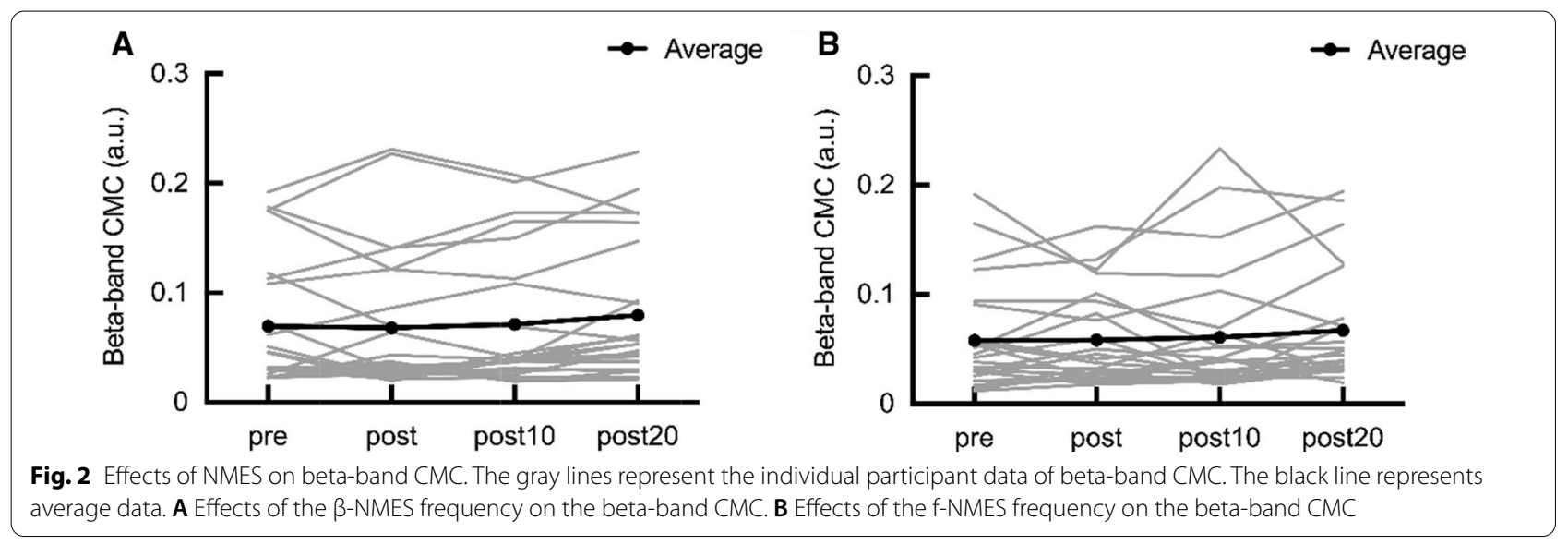



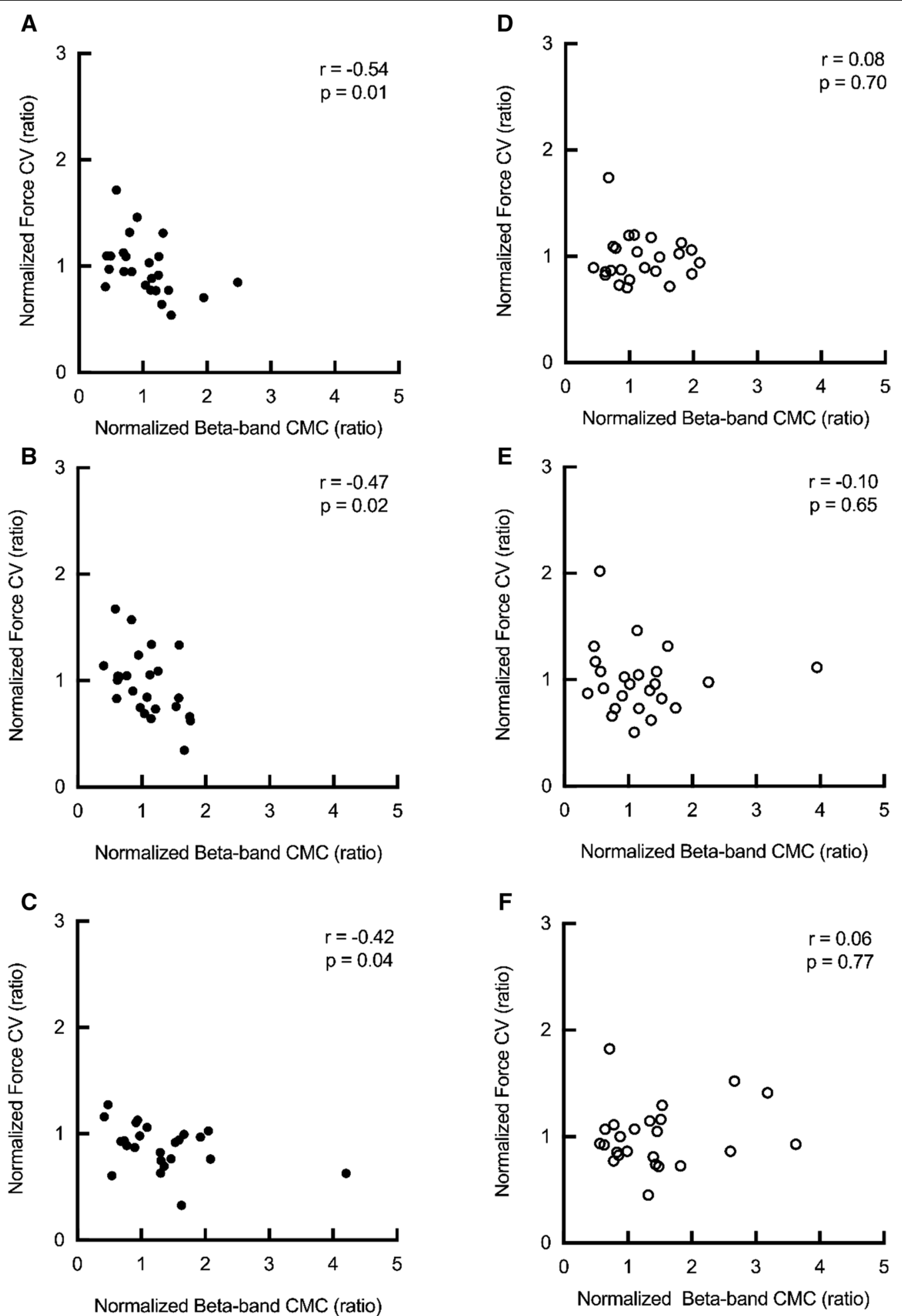

Fig. 3 Correlations between normalized beta-band CMC and normalized Force CV at each assessment time point. Data plots represent the relationships between the normalized beta-band CMC and normalized Force CV on the $\beta$-NMES (closed circle) at post (A), at post10 (B), and at post20 (C). Data plots represent that relationships between the normalized beta-band CMC and normalized Force CV on f-NMES (opened circle) at post $(\mathbf{D})$, at post10 (E), and at post20 (F) 
no significant correlations were found at post $(r=0.08$, $p=0.70$, Fig. $3 \mathrm{D})$, post $10(\mathrm{r}=-0.10, \mathrm{p}=0.65$, Fig. $3 \mathrm{E})$, and post $20(r=0.06, p=0.77$, Fig. 3F). These results indicate that changes in beta-band CMC following $\beta$-NMES are related with the changes in the exerted steady state of ankle dorsiflexion, meaning that the relationship is modulated by NMES with a beta-band CMC frequency.

\section{Complementary experiment MEPs}

A significant main effect was observed on the normalized MEPs $(p=0.02)$. However, post-hoc analysis revealed no significant differences at each time point compared with data pre ( $p>0.05$, Fig. 4).

\section{Relationship between the normalized MEPs and normalized beta-band CMC}

Figure 5 shows data plots on the normalized MEPs and normalized beta-band CMC. There was a significant positive correlation at post $(\mathrm{r}=0.69, \mathrm{p}=0.01$, Fig. $5 \mathrm{~B})$, whereas no significant correlations were observed at pre $(r=-0.42, p=0.89$, Fig. $5 A)$, at post $10(r=-0.15$, $\mathrm{p}=0.60$, Fig. $5 \mathrm{C})$, and at post $20(\mathrm{r}=0.27, \mathrm{p}=0.35$, Fig. 5D). The results indicated that changes in corticospinal excitability were related with data in beta-band CMC following $\beta$-NMES.

\section{Discussion}

The present study demonstrated no significant changes in the mean data of beta-band CMC, Force CV, and normalized MEPs in both the $\beta$-NMES and f-NMES cases. Whereas, NMES with beta-band CMC frequency affects changes in synchronization between the motor cortex and spinal motor neurons that correlate with changes in the exerted steady-state of isometric ankle dorsiflexion and changes in corticospinal excitability. These

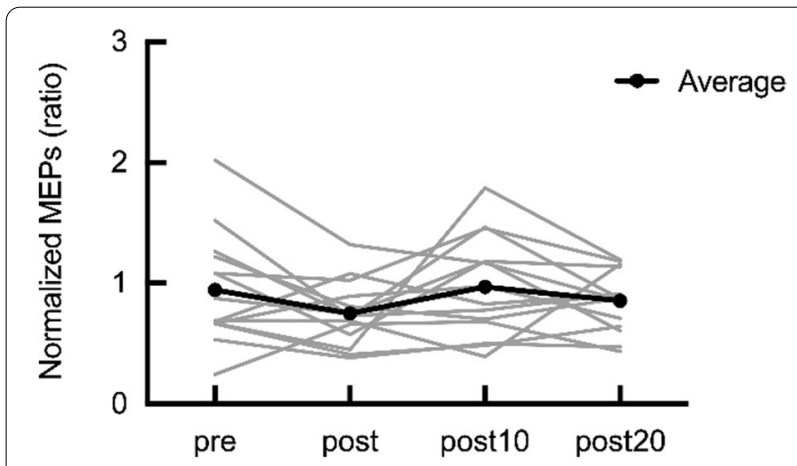

Fig. 4 Effects of the $\beta$-NMES on the motor evoked potentials (MEPs) of the tibialis anterior muscle. The gray lines represent the individual participant data with normalized MEP amplitude. The black line represents average data results may lead to the understanding of the mechanism underlying the sensory input via the beta-band frequency NMES based on physiological activity effects on corticospinal excitability and voluntary motor output. This suggestion may contribute to the development of effective NMES parameters for a neurorehabilitation in patients with motor dysfunction.

We expected that the $\beta$-NMES enhances the betaband $\mathrm{CMC}$, Force $\mathrm{CV}$, and normalized MEPs, but the results were unexpected. In the present study, $\beta$-NMES enhanced the beta-band CMC in 13 of the 24 participants $(54 \%)$. One possibility for these unexpected results is that there is variability of the effects of $\beta$-NMES on CMC [22, 23, 34-36]. A review paper regarding NMES parameters suggested that the intervention duration and stimulation intensity of NMES are important parameters for the modulation of the corticospinal excitability [34]. We set the duration of NMES to $20 \mathrm{~min}$ based on a previous study that has reported that the MEPs increased following 20 min of NMES [27]. Further consideration of the fact that the CMC changes were induced by muscle fatigue [37, 38], NMES with $95 \%$ of the motor threshold was set in this study. A study has reported that NMES with an intensity of below the motor threshold activated the cutaneous and sensory fibers and increased corticospinal excitabilities [39, 40]. However, 20-min NMES with an intensity of below the motor threshold might be insufficient to affect CMC and corticospinal excitability in this study. Indeed, a previous study has reported that an intensity below the motor threshold and/or a frequency of $100 \mathrm{~Hz}$ do not enhance the beta-band CMC [23]. Therefore, future research is warranted to investigate the effects of NMES with different intervention durations and stimulation intensities on CMC.

Conversely, the correlation between changes in betaband CMC and changes in Force CV were observed continually at least $20 \mathrm{~min}$. Previous studies reported that the concentration of cortical oscillation to specific frequency (e.g., $20 \mathrm{~Hz}$ ) reduces the variability of common synaptic inputs, and motor units discharge and improve the exerted steady-state force [41-43]. Thus, changes in the exerted steady-state of isometric ankle dorsiflexion are affected by changes in control from the motor cortex to spinal motor neurons, that is, by changes in the synchronization between them. The changes in beta-band $\mathrm{CMC}$ reflected changes in descending drive from the primary motor cortex to spinal motor neurons. This is supported by our complementary experiment indicating the correlation between changes in beta-band CMC and changes in MEPs immediately after $\beta$-NMES. Another study reported that cortical oscillation may modulate the firing rate of motor cortical efferent commands [1]. The cortical oscillation desynchronizes immediately after 

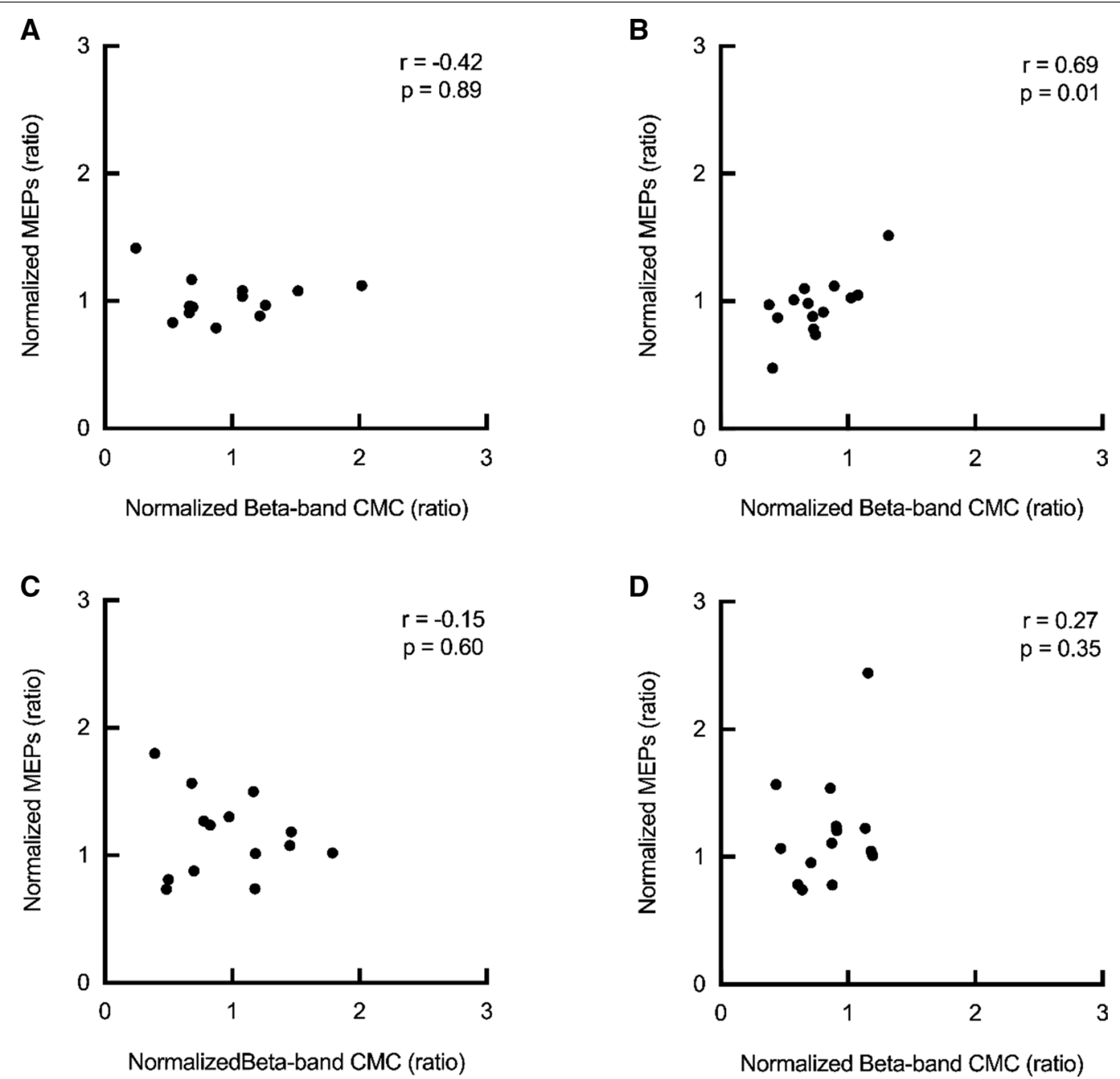

Fig. 5 Correlations between the normalized beta-band CMC and normalized MEPs at each assessment time point. Data plots represent that relationships between the normalized beta-band CMC and normalized MEPs on $\beta$-NMES at pre (A), post (B), post10 (C), and post20 (D)

the afferent input via the electrical stimulation, and then rebounds to a more effective synchronization than before stimulus [44]. Thus, our results suggested that $\beta$-NMES alters the corticospinal projections by inducing changes in the degree of interneuron's synchronization within the primary motor cortex. These physiological changes may affect the steady state of isometric ankle dorsiflexion. Alternatively, the correlation between changes in betaband CMC and those in Force CV was weak. Studies have reported that beta-band $\mathrm{CMC}$ was influenced by muscle fatigue and attention $[37,38,45,46]$. These factors might affect the results of this study [47].

In contrast, the correlation between changes in betaband $\mathrm{CMC}$ and changes in Force $\mathrm{CV}$ was not observed after f-NMES at a frequency of $100 \mathrm{~Hz}$. The f-NMES was set to emulate burst stimulation based on the sensory feedback from TA muscle spindles induced owing to the synaptic plasticity of the spinal interneuron circuit [26]. However, no changes were observed in corticospinal excitability in their study. Our complementary experiment observed the correlation between changes in beta-band CMC and changes in MEPs. Thus, the changes in the corticospinal excitability were responsible for the correlation between changes in beta-band $\mathrm{CMC}$ and changes in Force $\mathrm{CV}$ and not the plasticity in spinal interneuron.

The effects of fixed frequency within the beta-band (i.e., $20 \mathrm{~Hz}$ ) of NMES were not investigated in the present study. Additional studies are needed to (a) investigate the effect of fixed frequency within the beta-band of NMES, and (b) identify more effective stimulation time and intensity to enhance the mean data on beta-band CMC, Force CV, and MEPs. This study was conducted on healthy participants. Future studies are needed to 
test the current approach in patients with sensorimotor dysfunction.

\section{Conclusion}

The present study shows that NMES with beta-band $\mathrm{CMC}$ frequency was inadequate to enhance $\mathrm{CMC}$, Force $\mathrm{CV}$, and MEPs. However, $\beta$-NMES affects the relationship between changes in beta-band $\mathrm{CMC}$, Force $\mathrm{CV}$, and MEPs. We proposed that sensory input based on individual beta-band CMC frequencies may affect the relationship between beta-band physiological activity and voluntary motor output. Additional experiments are required to investigate the influence of the intervention duration and stimulation intensity.

\begin{abstract}
Abbreviations
aMT: Active motor threshold; $\beta$-NMES: Beta-band frequency neuromuscular electrical stimulation; CPN: Common peroneal nerve; CMC: Corticomuscular coherence; EEG: Electroencephalographic; EMG: Electromyographic; Force CV: Coefficient of variation of force; f-NMES: Fixed frequency neuromuscular electrical stimulation; MEPs: Motor evoked potentials; MVC: Maximum voluntary contraction; NMES: Neuromuscular electrical stimulation; TA: Tibialis anterior; tACS: Transcranial alternating current stimulation.
\end{abstract}

\section{Acknowledgements}

Not applicable.

\section{Authors' contributions}

TK and TY conceived and designed the experiments. TK, DK, and NK recruited participants. TK collected data and analyzed data. TK, DK, NK, SN, MN, and TY interpreted the results of experiments. ST constructed the program for data collection. TK and TY drafted the manuscript. All authors have read and approved the final manuscript.

\section{Funding}

This work was partially supported by grants from the Funds for a Grant-inAid for Young Scientists (18K17723) to Tomofumi Yamaguchi. Authors have used the grant for acquiring the experimental materials. The founder was not involved in the design, method, data collection and analysis, interpretation of data, and writing the manuscript.

\section{Availability of data and materials}

The datasets used and/or analyzed in the current study are available from the corresponding author on reasonable request.

\section{Declarations}

\section{Ethics approval and consent to participate}

This study was approved by the Ethics Review Board of Yamagata Prefectural University of Health Sciences (Approval number: 1806-06) and was performed in accordance with the ethical standards established by the Declaration of Helsinki. All participants provided their written informed consents before participating in the study.

\section{Consent to publication}

All participants provided their written informed consents for publication of the results before participating in the study.

\section{Competing interests}

The authors declare that they have no competing interest.

\section{Author details}

${ }^{1}$ Department of Physical Therapy, Yamagata Prefectural University of Health Sciences, 260 Kamiyanagi, Yamagata 990-2212, Japan. ${ }^{2}$ Department of Anatomy and Structural Science, Yamagata University School of Medicine, 2-2-2 lida-Nishi, Yamagata 990-9585, Japan. ${ }^{3}$ Faculty of Rehabilitation, School of Health Sciences, Fujita Health University, 1-98 Dengakugakubo, Kutsukake-cho, Toyoake, Aichi 470-1192, Japan. ${ }^{4}$ Department of Physical Therapy, Faculty of Health Science, Juntendo University, 2-1-1 Hongo, Bunkyo-ku, Tokyo 113-8421, Japan.

Received: 13 January 2021 Accepted: 1 October 2021

Published online: 13 October 2021

\section{References}

1. Salenius SPK, Kajola M, Salmelin R, Hari R. Cortical control of human motoneuron firing during isometric contraction. J Neurophysiol. 1997;77:3401-5.

2. Gross JTP, Salenius S, Hari R, Freund HJ, Schnitzler A. Corticomuscular synchronization during isometric muscle contraction in humans as revealed by magnetoencephalography. J Physiol. 2000;527:623-31.

3. Ushiyama J, Yamada J, Liu M, Ushiba J. Individual difference in beta-band corticomuscular coherence and its relation to force steadiness during isometric voluntary ankle dorsiflexion in healthy humans. Clin Neurophysiol. 2017;128:303-11.

4. Conway BA, Halliday DM, Farmer SF, Shahani U, Maas P, Weir Al, Rosenberg JR. Synchronization between motor cortex and spinal motoneuronal pool during the performance of a maintained motor task in man. J Physiol. 1995;489:917-24.

5. Baker SN, Lemon RN. Synchronization in monkey motor cortex during a precision grip task. II. Effect of oscillatory activity on corticospinal output. J Neurophysiol. 2003;89:1941-53.

6. Riddle CN, Baker SN. Manipulation of peripheral neural feedback loops alters human corticomuscular coherence. J Physiol. 2005;566:625-39.

7. Perez MA, Lundbye-Jensen J, Nielsen JB. Changes in corticospinal drive to spinal motoneurones following visuo-motor skill learning in humans. J Physiol. 2006;573:843-55.

8. Witham CL, Riddle CN, Baker MR, Baker SN. Contributions of descending and ascending pathways to corticomuscular coherence in humans. J Physiol. 2011;589:3789-800.

9. Mima THM. Corticomuscular coherence: a review. J Clin Neurophysiol. 1999;16:501-11.

10. Fang Y, Daly JJ, Sun J, Hvorat K, Fredrickson E, Pundik S, Sahgal V, Yue GH. Functional corticomuscular connection during reaching is weakened following stroke. Clin Neurophysiol. 2009;120:994-1002.

11. Kasuga S, Momose N, Ushiyama J, Ushiba J. Corticomuscular coherence reflects somatosensory feedback gains during motor adaptation. Neurosci Res. 2018;131:10-8.

12. Krauth R, Schwertner J, Vogt S, Lindquist S, Sailer M, Sickert A, Lamprecht J, Perdikis S, Corbet T, Millan JDR, et al. Cortico-muscular coherence is reduced acutely post-stroke and increases bilaterally during motor recovery: a pilot study. Front Neurol. 2019;10:126.

13. Fisher RJ, Galea MP, Brown P, Lemon RN. Digital nerve anaesthesia decreases EMG-EMG coherence in a human precision grip task. Exp Brain Res. 2002;145:207-14.

14. Phoja MSS, Hari R. Cortico-muscular coupling in a human subject with mirror movements-a magnetoencephalographic study. Neurosci Lett. 2002;327:185-8.

15. Kilner JMFR, Lemon RN. Coupling of oscillatory activity between muscles is strikingly reduced in a deafferented subject compared with normal controls. J Neurophysiol. 2004;92:790-6.

16. Baker SN, Chiu M, Fetz EE. Afferent encoding of central oscillations in the monkey arm. J Neurophysiol. 2006;95:3904-10.

17. Korvenoja A, Huttunen J, Salli E, Pohjonen H, Martinkauppi S, Palva JM, Lauronen L, Virtanen J, Ilmoniemi and RJ, Aronen HJ. . Activation of multiple cortical areas in response to somatosensory stimulation: combined magnetoencephalographic and functional magnetic resonance imaging. Hum Brain Mapp. 1999;8:13-27.

18. Nihashi T, Naganawa S, Sato C, Kawai H, Nakamura T, Fukatsu H, Ishigaki T, Aoki I. Contralateral and ipsilateral responses in primary somatosensory cortex following electrical median nerve stimulation-an fMRI study. Clin Neurophysiol. 2005;116:842-8. 
19. Carson RG, Buick AR. Neuromuscular electrical stimulation-promoted plasticity of the human brain. J Physiol. 2021;599(9):2375-99.

20. Schabrun SM, Ridding MC, Galea MP, Hodges PW, Chipchase LS. Primary sensory and motor cortex excitability are co-modulated in response to peripheral electrical nerve stimulation. PLOS ONE. 2012;7: e51298.

21. Yamaguchi T, Sugawara K, Tanaka S, Yoshida N, Saito K, Tanabe S, Muraoka Y, Liu M. Real-time changes in corticospinal excitability during voluntary contraction with concurrent electrical stimulation. PLOS ONE. 2012;7: e46122.

22. Pan LH, Yang WW, Kao CL, Tsai MW, Wei SH, Fregni F, Chen VC, Chou LW. Effects of 8-week sensory electrical stimulation combined with motor training on EEG-EMG coherence and motor function in individuals with stroke. Sci Rep. 2018;8(1):9217.

23. Lai MI, Pan LL, Tsai MW, Shih YF, Wei SH, Chou LW. Investigating the effects of peripheral electrical stimulation on corticomuscular functional connectivity stroke survivors. Top Stroke Rehabil. 2016;23:154-62.

24. Yamaguchi T, Svane C, Forman CR, Beck MM, Geertsen SS, LundbyeJensen J, Nielsen JB. Transcranial alternating current stimulation of the primary motor cortex after skill acquisition improves motor memory retention in humans: a double-blinded sham-controlled study. Cereb Cortex Commun. 2020;1:1-11.

25. Chapman JPCL, Allen JJ. The measurement of foot preference. Neuropsychologia. 1987;25:579-84.

26. Perez MA, Field-Fote EC, Floeter MK. Patterned sensory stimulation induces plasticity in reciprocal la inhibition in humans. J Neurosci. 2003;23:2014-8.

27. Andrews RKSS, Ridding MC, Gaela MP, Hodges PW, Chipchase LS. The effect of electrical stimulation on corticospinal excitability is dependent on application duration: a same subject pre-post test design. J Neuroeng Rehabil. 2013;10:51-7.

28. Spedden ME, Nielsen JB, Geertsen SS. Oscillatory corticospinal activity during static contraction of ankle muscles is reduced in healthy old versus young adults. Neural Plast. 2018:20:1-13.

29. Halliday DMRJ, Amjad AM, Breeze P, Conway BA, Farmer SF. A framework for the analysis of mixed time series/point process data-theory and application to the study of physiological tremor, single motor unit dis- charges and electromyograms. Prog Biophys Mol Biol. 1995;64:237-78.

30. Keil J, Timm J, Sanmiguel I, Schulz H, Obleser J, Schonwiesner M. Cortical brain states and corticospinal synchronization influence TMS-evoked motor potentials. J Neurophysiol. 2014;111:513-9.

31. Khaslavskaia S, Ladouceur M, Sinkjaer T. Increase in tibialis anterior motor cortex excitability following repetitive electrical stimulation of the common peroneal nerve. Exp Brain Res. 2002;145:309-15.

32. Knash ME, Kido A, Gorassini M, Chan KM, Stein RB. Electrical stimulation of the human common peroneal nerve elicits lasting facilitation of cortical motor-evoked potentials. Exp Brain Res. 2003;153:366-77.

33. Mang CS, Lagerquist $O$, Collins DF. Changes in corticospinal excitability evoked by common peroneal nerve stimulation depend on stimulation frequency. Exp Brain Res. 2010;203:11-20.
34. Chipchase LS, Schabrun SM, Hodges PW. Peripheral electrical stimulation to induce cortical plasticity: a systematic review of stimulus parameters. Clin Neurophysiol. 2011;122:456-63.

35. McClelland VM, Cvetkovic Z, Mills KR. Modulation of corticomuscular coherence by peripheral stimuli. Exp Brain Res. 2012;219:275-92.

36. Xu R, Wang Y, Wang K, Zhang S, He C, Ming D. Increased corticomuscular coherence and brain activation immediately after short-term neuromuscular electrical stimulation. Front Neurol. 2018;9:886.

37. Yang Q, Fang Y, Sun C-K, Siemionow V, Ranganathan VK, Khoshknabi D, Davis MP, Walsh D, Sahgal V, Yue GH. Weakening of functional corticomuscular coupling during muscle fatigue. Brain Res. 2009;1250:101-12.

38. Ushiyama J, Katsu M, Masakado Y, Kimura A, Liu M, Ushiba J. Muscle fatigue-induced enhancement of corticomuscular coherence following sustained submaximal isometric contraction of the tibialis anterior muscle. J Appl Physiol. 2011;110:1233-40.

39. Hamdy SR, Rothwell JC, Aziz Q, Singh KD, Thompson DG. (1998) Longterm reorganization of human motor cortex driven by short-term sensory stimulation. Nat Neurosci. 1998;1:64-8.

40. Kaelin-Lang A, Luft AR, Sawaki L, Burstein AH, Sohn YH, Cohen LG. Modulation of human corticomotor excitability by somatosensory input. J Physiol. 2002;540:623-33.

41. Kouzaki M, Kimura T, Yoshitake Y, Hayashi T, Moritani T. Subthreshold electrical stimulation reduces motor unit discharge variability and decreases the force fluctuations of plantar flexion. Neurosci Lett. 2012;513:146-50.

42. Watanabe RN, Kohn AF. Fast oscillatory commands from the motor cortex can be decoded by the spinal cord for force control. J Neurosci. 2015;35:13687-97.

43. Feeney DF, Mani D, Enoka RM. Variability in common synaptic input to motor neurons modulates both force steadiness and pegboard time in young and older adults. J Physiol. 2018;596:3793-806.

44. Neuper CPG. Evidence for distinct beta resonance frequencies in human EEG related to specific sensorimotor cortical areas. Clin Neurophysiol. 2001;112:2084-97.

45. Mat Safri N, Murayama N, Hayashida Y, Igasaki T. Effects of concurrent visual tasks on cortico-muscular synchronization in humans. Brain Res. 2007;1155:81-92.

46. Johnson AN, Wheaton LA, Shinohara M. Attenuation of corticomuscular coherence with additional motor or non-motor task. Clin Neurophysiol. 2011;122:356-63.

47. Gorgey AS, Black CD, Elder CP, Dudley GA. Effects of electrical stimulation parameters on fatigue in skeletal muscle. J Orthop Sports Phys Ther. 2009;39:684-92.

\section{Publisher's Note}

Springer Nature remains neutral with regard to jurisdictional claims in published maps and institutional affiliations.

Ready to submit your research? Choose BMC and benefit from:

- fast, convenient online submission

- thorough peer review by experienced researchers in your field

- rapid publication on acceptance

- support for research data, including large and complex data types

- gold Open Access which fosters wider collaboration and increased citations

- maximum visibility for your research: over 100M website views per year

At BMC, research is always in progress.

Learn more biomedcentral.com/submissions 